\title{
Influência do Grau de Substituição e da Distribuição de Substituintes Sobre as Propriedades de Equilíbrio de Carboximetilcelulose em Solução Aquosa
}

\author{
José Cláudio Caraschi e Sérgio P. Campana Filho
}

Resumo: Amostras de carboximetilcelulose foram preparadas por reação heterogênea de polpa soda/antraquinona obtida de fibras pré-hidrolisadas de bagaço de cana-de-açúcar. As amostras com os menores graus de substituição $(\overline{G S}<1,0)$ e as mais substituídas $(\overline{G S}>1,0)$ apresentaram comportamentos diferentes quanto a: 1$)$ variação de constante aparente de acidez ( $\mathrm{pk}_{\mathrm{ap}}$ ) com o grau de dissociação; ii) concordância entre os valores de $\overline{G S}$ determinados por titulações e por espectroscopia ${ }^{1} \mathrm{H}$ rmn e iii) concordância entre os valores experimentais de coeficientes de transporte e os previstos pelo modelo de Manning. Todas as amostras apresentaram um padrão de substituição em blocos que, nas amostras menos substituídas são curtos e espaçados, favorecendo interações associativas que levam à agregação. Efeitos de volume excluído eletrostático, mais importantes nas amostras mais substituídas, inibem a agregação e explicam o comportamento observado nesses casos.

Palavras-chave: Carboximetilcelulose, grau de substituição, constante de acidez, coeficiente de transporte

\section{Introdução}

Dentre os produtos obtidos através de modificações químicas de celulose, a carboximetilcelulose (CMC) é um dos que mais se destaca em função da sua importância econômica como agente espessante e pela variedade de aplicações ${ }^{[1,2]}$. Carboximetilcelulose é um derivado hidrossolúvel obtido industrialmente a partir da reação em suspensão ("slurry process") de celulose, hidróxido de sódio e ácido monocloroacético. Este derivado é, em geral, preparado e utilizado na forma sódica ( $\mathrm{CMCNa}$ ) que, uma vez dissolvido em água, apresenta as características típicas de polieletrólitos. Suas propriedades e aplicações dependem, essencialmente, da viscosidade de suas soluções aquo- sas, do grau médio de substituição $(\overline{G S})$, definido como o número médio de grupos hidroxila substituídos por unidade D-glicopiranosil da cadeia polimérica, e da distribuição dos grupos carboximetila. Este último fator, a distribuição dos grupos carboximetila ao longo das cadeias, influencia fortemente suas propriedades, o comportamento reológico de suas soluções e a abrangência de sua aplicações. Os produtos industrialmente importantes apresentam graus médios de substituição inseridos em uma faixa relativamente restrita $(0,5<\overline{G S}<1,5)$ e, considerando que são obtidos por reação heterogênea, a distribuição dos substituintes inseridos nas cadeias de celulose depende da acessibilidade dos reagentes aos sítios reativos da macromolécula, sendo uma característica de difícil controle. 

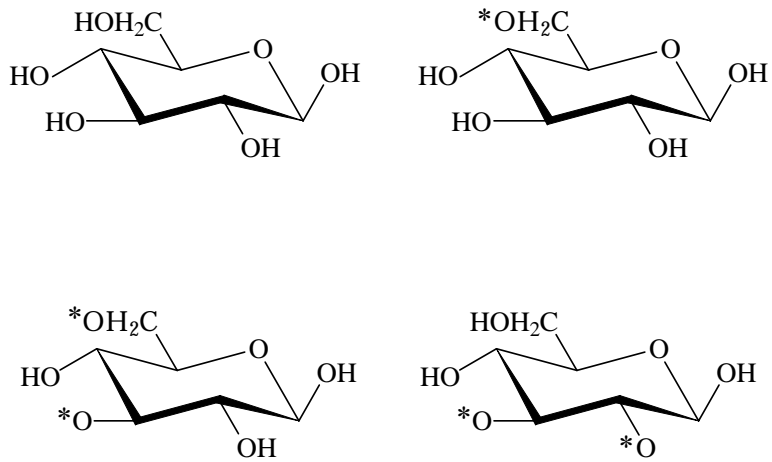
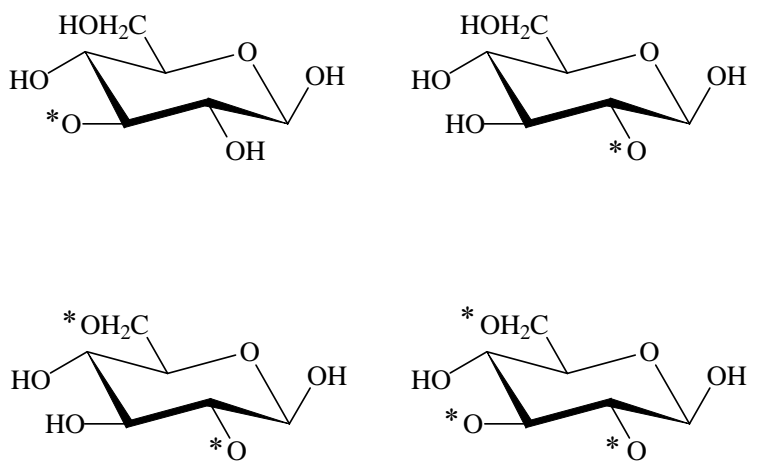

Figura 1. Estruturas das oito possíveis unidades repetitivas presentes nas cadeias de carboximetilcelulose e correspondentes a introdução de até três grupos carboximetila por resíduo de glicose. $\mathrm{O}$ símbolo (*) identifica as posições em que o grupo $-\mathrm{CH}_{2} \mathrm{COO}^{-} \mathrm{Na}^{+}\left(\right.$ou $\left.-\mathrm{CH}_{2} \mathrm{COOH}\right)$ foi introduzido.

De modo geral, as amostras de carboximetilcelulose obtidas através da reação heterogênea são constituídas por cadeias que podem conter até oito unidades repetitivas diferentes (Figura 1).

A preparação de $\mathrm{CMC}$ por reação heterogênea de carboximetilação de celulose resulta em produtos cuja distribuição de substituintes ocorre em blocos, nos quais os grupos carboximetila são mais freqüentes nas posições 2 e 6 dos resíduos de glicopiranose ${ }^{[3-6]}$. Por outro lado, distribuições mais uniformes de substituintes são obtidas através da carboximetilação em meio homogêneo ${ }^{[3,7]}$, por reação heterogênea à medida que $\overline{G S}$ aumenta ${ }^{[3,4]}$, e perfeitamente regulares quando a cadeia celulósica é completamente substituída $(\overline{G S}=3)$.

Apenas nesse último caso soluções molecularmente dispersas de derivados de celulose podem ser obtidas sendo que, incluso o caso de CMC de baixo grau de substituição $(\overline{G S}<1)$ dispersa em meio aquoso ${ }^{[6]}$, amostras parcialmente substituídas levam à formação de estruturas agregadas ou autoassociadas ${ }^{[6,8]}$.

Assim, a distribuição de substituintes ao longo das cadeias poliméricas e a presença de agregados em solução podem afetar as caracterizações de CMC e influenciar suas propriedades de equilíbrio.

Para avaliar a influência do grau médio de substituição e da distribuição dos substituintes carboximetila sobre as propriedades de CMC, as amostras deste trabalho são caracterizadas quanto a graus médios de substituição determinados por duas técnicas indepen- dentes e são analisadas quanto a constantes intrínsecas de acidez.

Com os objetivos de avaliar a ocorrência de distribuições uniformes de substituintes em carboximetilcelulose e sua influência sobre as propriedades do polímero, este trabalho também emprega o modelo de Manning ${ }^{[9]}$ para calcular valores de coeficientes de transporte das cadeias poliméricas e compara essas previsões com os valores determinados experimentalmente através de titulações condutimétricas.

\section{Experimental}

\section{Obtenção e purificação das amostras de carboximetilcelulose}

As amostras de carboximetilcelulose foram preparadas em meio heterogêneo pelo processo em suspensão de isopropanol/água, empregando a polpa branqueada soda/AQ de fibras pré-hidrolisadas de bagaço de cana-de-açúcar como fonte de celulose ${ }^{[10,11]}$. Os sítios reativos nas unidades de glicopiranose são as hidroxilas ligadas aos carbonos 2, 3 e 6 (Figura 2).

As purificações das amostras de $\mathrm{CMC}$ foram realizadas através de procedimento experimental que consiste em dissolução da amostra em meio aquoso contendo excesso de $\mathrm{NaCl}$, filtração da solução resultante e adição de etanol absoluto até precipitação, seguida de lavagens com misturas etanol/água para eliminação de excesso de sal. Após a execução dessa
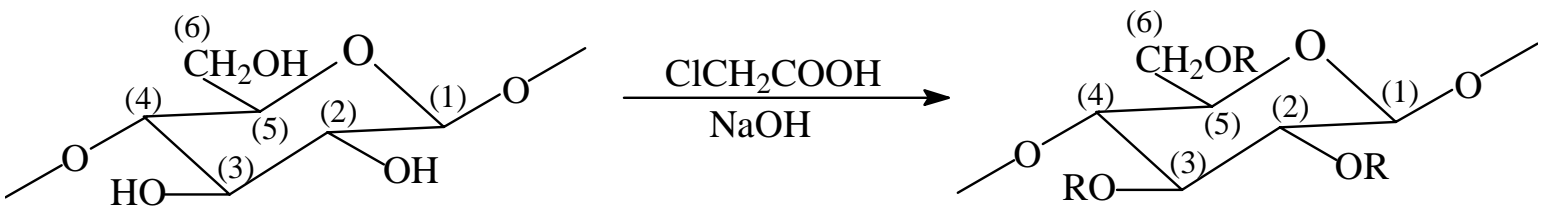

Figura 2. Representação esquemática da introdução do grupo carboximetila no anel de glicopiranose, com $\mathrm{R}=\mathrm{H}$ ou $\mathrm{CH}_{2} \mathrm{COO} \mathrm{Na}^{+}$. 
etapa, amostras purificadas na forma sódica $(\mathrm{CMCNa})$ foram obtidas como pós brancos que são fácil e completamente dispersos em meio aquoso ${ }^{[10]}$.

\section{Caracterizações}

As amostras purificadas de carboximetilcelulose foram caracterizadas quanto a graus médios de substituição $(\overline{G S})$, coeficientes de transporte (f) e constantes de acidez intrínseca $\left(\mathrm{pk}_{0}\right)$, conforme descrito a seguir.

Graus de substituição, coeficientes de transporte e constantes de acidez

Os graus médios de substituição $(\overline{G S})$ das amostras de CMC foram determinados por espectroscopia de ressonância magnética nuclear do próton ${ }^{[12]}$ e por titulações condutimétricas e potenciométricas ${ }^{[13]}$.

No primeiro caso, após hidrólise da amostra em $\mathrm{D}_{2} \mathrm{O} / \mathrm{D}_{2} \mathrm{SO}_{4}(2: 1 \mathrm{v} / \mathrm{v})$, ácido acético glacial foi adicionado como referência interna e uma alíquota foi transferida para o tubo adequado à aquisição do espectro em equipamento Brucker 200. Os valores de graus de substituição foram obtidos através da razão entre as integrais dos prótons metilênicos dos grupos carboximetila e as integrais dos prótons pertencentes ao anel da unidade $\beta$-D-glicopiranosil ligados aos carbonos C2, C3, C4, C5 e C6 (Figura 2), empregando a seguinte expressão:

$$
(\overline{\mathrm{GS}})=\frac{\mathrm{a} / 2}{\mathrm{~b} / 6}
$$

onde: $\mathrm{a}=$ integral referente aos prótons metilênicos da carboximetila $(3,8 \mathrm{ppm}<\delta<4,3 \mathrm{ppm}) ; \mathrm{b}=$ integral referente aos 6 prótons ligados ao anel da unidade $\beta$-D-glicopiranosil $(2,8<\delta<3,8 \mathrm{ppm})$.

As titulações condutimétricas e potenciométricas das soluções de CMC com bases fortes foram realizadas após a conversão das amostras de CMC em suas respectivas formas ácidas por passagem em coluna de troca de cátions Amberlite IR-120H, condicionada na forma ácida. As amostras de $\mathrm{CMCNa}$ foram dissolvidas em água deionizada após 18 horas de agitação a temperatura ambiente, as concentrações finais das soluções de $\mathrm{CMCH}$ empregadas nas titulações foram de aproximadamente $1 \mathrm{~g} / \mathrm{L}$ e os valores de $\overline{G S}$ foram calculados a partir da seguinte expressão:

$$
\overline{\mathrm{GS}}=\frac{162 \mathrm{E}}{\mathrm{P}-80 \mathrm{E}}
$$

onde: $\mathrm{P}=$ massa em gramas de amostra de $\mathrm{CMC}$; $\mathrm{E}=$ número de equivalentes de base utilizada em equivalentes/L.

A partir das curvas de titulações condutimétricas das amostras de carboximetilcelulose na forma ácida com $\mathrm{LiOH}$ e $\mathrm{KOH}$, os coeficientes de transporte $\left(\mathrm{f}_{\text {exp. }}\right)$ foram calculados pela expressão abaixo:

$$
\mathrm{f}_{\mathrm{exp}}=\frac{\left(\sigma_{\mathrm{K}^{+}}-\sigma_{\mathrm{Li}^{+}}\right)_{\mathrm{eq}} 10^{-3}}{\mathrm{C}_{\mathrm{P}}\left(\lambda_{\mathrm{K}^{+}}-\lambda_{\mathrm{Li}^{+}}\right)}
$$

onde: $\sigma_{\text {eq }}=$ condutância $\left(\Omega^{-1} \mathrm{~cm}^{2}\right)$ da solução no ponto de equivalência, o subíndice $\left(\mathrm{K}^{+}\right.$ou $\left.\mathrm{Li}^{+}\right)$indicando o cátion metálico presente na base utilizada na titulação; $\mathrm{C}_{\mathrm{P}}=$ concentração (eq/L) da solução de carboximetilcelulose na forma ácida; $\lambda=$ condutância equivalente $\left(\Omega^{-1} \mathrm{~cm}^{2} \mathrm{eq}^{-1}\right)$ do contra-íon, o subíndice indicando o contra-íon metálico presente na base utilizada na titulação.

O modelo de Manning ${ }^{[9]}$ foi empregado para os cálculos de coeficientes de transporte ( $f_{\text {Manning }}$ ) através das expressões abaixo, válidas para soluções diluídas de polieletrólitos em presença de contra-íons monovalentes:

para $\lambda<1$ vem: $\mathrm{f}_{\text {Manning }}=1-\frac{0,55 \lambda^{2}}{\lambda+3,14}$

para $\lambda>1$ vem: $\mathrm{f}_{\text {Manning }}=\frac{0,87}{\lambda}$

Nas expressões acima, $\lambda$ é a densidade linear de carga definida por Lifson-Katchalsky ${ }^{[14]}$ que, para carboximetilcelulose, pode ser expressa por:

$$
\lambda=\frac{\alpha \overline{\mathrm{GS}}}{\mathrm{b}} \frac{\mathrm{e}^{2}}{\mathrm{DkT}}
$$

onde: $\alpha=$ grau de dissociação; $G S \overline{\mathrm{b}}=$ número de grupos iônicos por unidade de comprimento; $\mathrm{e}=$ carga do elétron; $\mathrm{D}=$ constante dielétrica do meio; $\mathrm{k}=$ constante de Boltzman; $\mathrm{T}=$ temperatura absoluta.

Considerando que $\mathrm{CMCNa}$ dissolvido em água a $25^{\circ} \mathrm{C}$ está totalmente dissociado $(\alpha \rightarrow 1)$, e substituindo o valor de b na expressão (6) pelo comprimento da unidade repetitiva $\left(b=5,15\right.$ Angstrons), vem $^{[15]}$.

$$
\lambda=1,38 \overline{G S}
$$


A partir das curvas das titulações de CMC na forma ácida $(\mathrm{CMCH})$ com solução padronizada de $\mathrm{NaOH}$, as constantes aparentes de acidez das amostras $\left(\mathrm{pk}_{\mathrm{ap}}\right)$ são determinadas para cada etapa da neutralização através da expressão abaixo:

$$
\mathrm{pk}_{\mathrm{ap}}=\mathrm{pH}+\log \frac{1-\alpha}{\alpha}
$$

Nessa expressão, $\alpha$ corresponde ao grau total de dissociação do polieletrólito, considerando também a sua autodissociação a cada incremento de $\mathrm{pH}$, tal que:

$$
\alpha=\alpha_{\mathrm{N}}+\alpha_{\mathrm{H}}
$$

onde: $\alpha_{\mathrm{N}}=$ grau de neutralização do polieletrólito; $\alpha_{\mathrm{H}}=\left[\mathrm{H}^{+}\right] / \mathrm{C}_{\mathrm{p}}=$ grau de autodissociação do polieletrólito; $\mathrm{C}_{\mathrm{p}}=$ concentração do polieletrólito em $\mathrm{eq} / \mathrm{L}$.

Os valores de constantes intrínsecas de acidez $\left(\mathrm{pk}_{0}\right)$ são determinados a partir das curvas de $\mathrm{pk}_{\mathrm{ap}}$ versus $\alpha$, construídas através do emprego da expressão (8), por extrapolação à situação em que o primeiro grupo carboxílico está dissociado $(\alpha \rightarrow 0)$.

\section{Resultados e Discussões}

\section{Constantes de acidez, graus médios de substituição e distribuição de substituintes}

Diferentemente do que ocorre com ácidos fracos comuns, como ácido acético, as curvas de titulação de poliácidos fracos, como $\mathrm{CMCH}$, não apresentam a típica região tampão.

No caso de $\mathrm{CMCH}$, à medida que a cadeia polimérica vai portando progressivamente mais cargas negativas devido à reação de neutralização, energia adicional vai sendo necessária para separar os prótons remanescentes dos grupos iônicos da cadeia. Disso resulta que a constante aparente de acidez $\left(\mathrm{pk}_{\mathrm{ap}}\right)$ aumenta com a dissociação do poliácido (Figura 3). Esse efeito, decorrente da natureza polieletrolítica e independente do grau de polimerização, pode ser afetado pela densidade e pela distribuição de cargas nas cadeias $^{[16]}$.

$\mathrm{O}$ ajuste polinomial dos pontos experimentais resulta em valores de $\mathrm{pk}_{0}$ (Tabela 1 ) que são semelhantes a outros descritos na literatura ${ }^{[15,17]}$. Entretanto, embora os valores de $\mathrm{pk}_{0}$ sejam muito próximos entre si, ou seja, um efeito ligado à densidade de carga não é claramente observado, dois tipos distintos de comportamentos podem ser identificados (Figura 3),

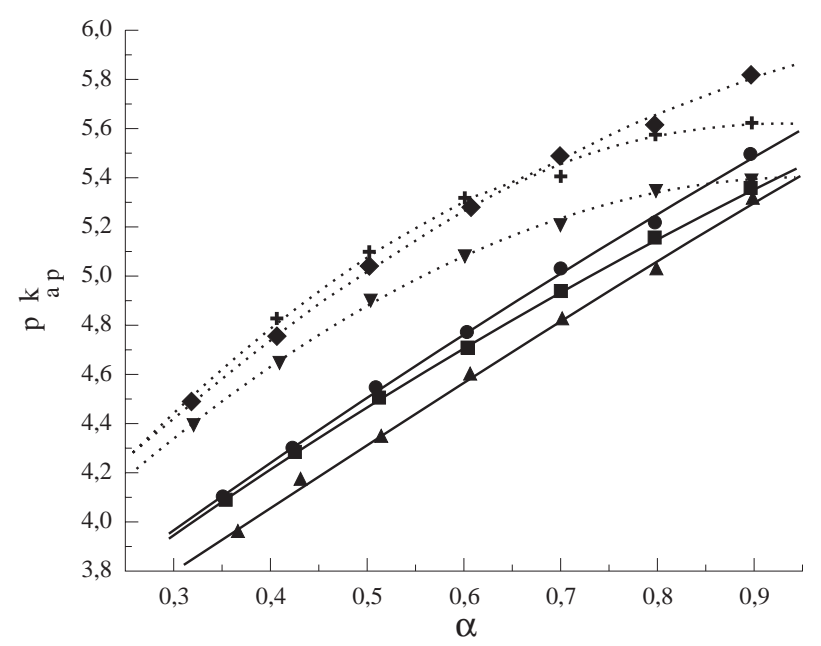

Figura 3. Curvas de constante aparente de acidez $\left(\mathrm{pk}_{\mathrm{a}}\right)$ versus grau total de dissociação $(\alpha)$ para as amostras de CMC: A (n); B (1); C (s); D (t); $\mathrm{E}(\mathrm{u}) ; \mathrm{F}(+)$.

correspondentes a amostras menos substituídas (curvas contínuas) e mais substituídas (curvas tracejadas).

Nas amostras menos substituídas, $\mathrm{pk}_{\mathrm{ap}}$ aumenta linearmente com o aumento do grau de dissociação do polieletrólito, o que sugere que os grupos acessíveis ao titulante estão relativamente distantes e regularmente espaçados. Tal possibilidade é sugerida pelas diferenças entre $\overline{G S}_{\text {tit }}$ e $\overline{G S}_{\text {rmn }}$ (Tabela 1), indicativas da presença de agregados, que tornam alguns grupos carboxílicos indetectáveis por titulação, e cuja formação é favorecida pela ocorrência de um padrão de substituição em blocos ${ }^{[8]}$ semelhante ao proposto acima.

As curvas das amostras mais substituídas podem ser subdivididas em dois segmentos lineares com inclinações diferentes. No segundo ramo dessas curvas $(\alpha>0,6)$, embora as variações de $\mathrm{pk}_{\mathrm{ap}}$ com o aumento de $\alpha$ sejam menores, a extrapolação desses segmentos de retas para a determinação dos valores de $\mathrm{pk}_{0}(\alpha \rightarrow 0)$, leva à conclusão que os grupos carboxílicos remanescentes são mais difíceis de serem dissociados $\left(4,3<\mathrm{pk}_{0}<4,7\right)$.

A extrapolação dos segmentos de retas do primeiro ramo $(\alpha<0,6)$ das curvas das amostras mais substituídas leva à conclusão que os primeiros $3 / 5$ dos seus grupos carboxílicos também são menos áci$\operatorname{dos}\left(\mathrm{pk}_{0} \cong 3,65\right)$ que os correspondentes das amostras menos substituídas $\left(3,0<\mathrm{pk}_{0}<3,2\right)$.

Nessa comparação entre os dois grupos de amostras, resulta aparente que o aumento do número de grupos carboximetila é responsável pelo aumento de $\mathrm{pk}_{0}$, mas outros resultados sugerem que a distribuição desses grupos também pode ter um papel muito importante. 
Tabela 1. Valores médios de graus de substituição $(\overline{G S})$ e de constantes intrínsecas de acidez $\left(\mathrm{pk}_{0}\right)$ das amostras de carboximetilcelulose.

\begin{tabular}{|c|c|c|c|}
\hline Amostras & {$\left[\overline{G S}_{\mathrm{RMN}}\right]^{(\mathrm{a})}$} & {$\left[\overline{G S}_{\mathrm{TIT}}\right]^{(\mathbf{b})}$} & {$\left[\mathrm{pk}_{0}\right]^{(\mathrm{c})}$} \\
\hline A (ם) & 0,82 & 0,67 & 3,07 \\
\hline $\mathrm{B}(\bullet)$ & 0,87 & 0,72 & 3,10 \\
\hline $\mathrm{C}(\bullet)$ & 1,00 & 0,89 & 3,02 \\
\hline $\mathrm{D}(\diamond)$ & 1,46 & 1,28 & 3,19 \\
\hline $\mathrm{E}(\diamond)$ & 1,65 & 1,54 & 3,35 \\
\hline $\mathrm{F}(+)$ & 1,78 & 1,71 & 3,20 \\
\hline
\end{tabular}

a) valores médios obtidos através de duas determinações independentes empregando espectroscopia ${ }^{1} \mathrm{H} \mathrm{rmn}$; b) valores médios obtidos de titulações independentes com soluções aquosas de $\mathrm{NaOH}$, LiOH e KOH; c) valores determinados pela extrapolação das curvas da Figura 1, sendo as amostras identificadas pelos símbolos da primeira coluna da tabela.

Assim, as determinações de composição e análises de ${ }^{1} \mathrm{H}$ rmn revelam que o número de unidades repetitivas dicarboximetiladas e tricarboximetiladas aumenta exponencialmente com o aumento do grau de substituição no intervalo considerado ${ }^{[3,4]}$, e que as posições 2 e 6 do anel glicopiranosídico são mais favorecidas para substituição ${ }^{[3,4,18]}$.

Tal padrão de substituição, em que a proximidade entre grupos substituintes é maior, pode facilitar as interações entre grupos carboxílicos de unidades adjacentes e dificultar a sua dissociação, o que justificaria as diferenças observadas entre amostras mais e menos substituídas de CMC deste trabalho.

De fato, a dissociação de poliácidos dicarboxílicos, tais como poli(ácido fumárico) e poli(ácido maleíco), parece ser um processo em duas etapas em que grupos com diferentes constantes de acidez são ionizados $^{[19]}$. Nesses casos, a proximidade entre grupos carboximetila é maior e o efeito de suas interações sobre $\mathrm{pk}_{0}$ são mais importantes.

A comparação entre os valores médios de graus de substituição determinados por espectroscopia de ressonância magnética nuclear e através de titulações, também revela diferenças entre as amostras mais e menos substituídas.

Para as amostras menos substituídas o emprego de ${ }^{1} \mathrm{H}$ rmn resulta em valores de $\overline{G S}$ até $20 \%$ maiores que os determinados por titulações. Por outro lado, a concordância entre os valores determinados pelas duas técnicas é melhor quanto maior o valor de $\overline{G S}$ (Tabela 1 ).

Este padrão já foi descrito na literatura e sua ocorrência atribuída a processos de agregação, mais favorecidos no caso das amostras menos substituídas, que afetam as titulações, mas não as determinações espectroscópicas de $\overline{G S}{ }^{[12]}$.

De fato, nas determinações de $\overline{G S}$ por ${ }^{1} \mathrm{H}$ rmn as amostras são previamente hidrolisadas e todas as unidades das cadeias de CMC sujeitas à análise espectroscópica, sem qualquer interferência ligada ao caráter macromolecular e ao comportamento associativo do polímero e, assim, os seus valores de $\overline{G S}$ são maiores.

Por outro lado, o tratamento para converter $\mathrm{CMCNa}$ (forma sódica) em sua forma ácida $(\mathrm{CMCH})$, que é realizado antes das titulações potenciométricas e condutimétricas, pode favorecer a agregação macromolecular em solução.

Evidência nesse sentido foi relatada em estudo recente sobre a distribuição de substituintes, no qual a lactonização envolvendo grupos carboxila e hidroxila de CMC $(0,76<\overline{G S}<2,24)$ foi observada após o tratamento para conversão das amostras à forma ácida, resultando em efetivo intercruzamento ${ }^{[5]}$. Além disso, amostras de CMC com $\overline{G S}<1,0$, preparadas na presença de excesso de ácido monocloroacético, são substancialmente insolúveis em meio aquoso neutro, o que pode ser atribuído à ocorrência de interações, por ligações de hidrogênio ou de intercruzamentos covalentes, envolvendo os grupos hidroxila e carboximetila de $\mathrm{CMCH}^{[20]}$. A conversão dos grupos carboxilato às suas formas ácidas resulta em considerável diminuição da densidade linear de carga das cadeias macromoleculares na solução aquosa diluída ( $\mathrm{pH} \cong 3,0)$ obtida após o tratamento. De fato, a partir dos valores de $\mathrm{pk}_{0}$ das amostras de CMC (Tabela 1), pode ser estimado que, em todos os casos, no máximo $50 \%$ dos grupos carboxílicos estão ionizados em solução aquosa diluída de $\mathrm{pH} \cong 3,0$. Nessa situação o enovelamento das cadeias poliméricas e também a ocorrência de agregação são mais acentuadamente favorecidos nos casos das amostras menos substituídas, pois interações repulsivas de natureza eletrostática, que contribuem para a expansão das cadeias, são menos importantes nesses casos. Nas amostras mais substituídas a densidade de carga é maior e, na mesma situação discutida acima ( $\mathrm{pH} \cong 3,0)$, as interações eletrostáticas são mais importantes, contribuindo para expandir o novelo polimérico e, por conseqüência, desfavorecendo processos associativos.

À medida que mais cargas vão sendo introduzidas nas cadeias de celulose, como ocorre na preparação de amostras mais substituídas de CMC, os efeitos eletrostáticos se tornam mais importantes, os processos de agregação são menos favorecidos e o efeito do caráter macromolecular e do comportamento associativo do polímero sobre as determinações de $\overline{G S}$ por titulações é paulatinamente reduzido. 


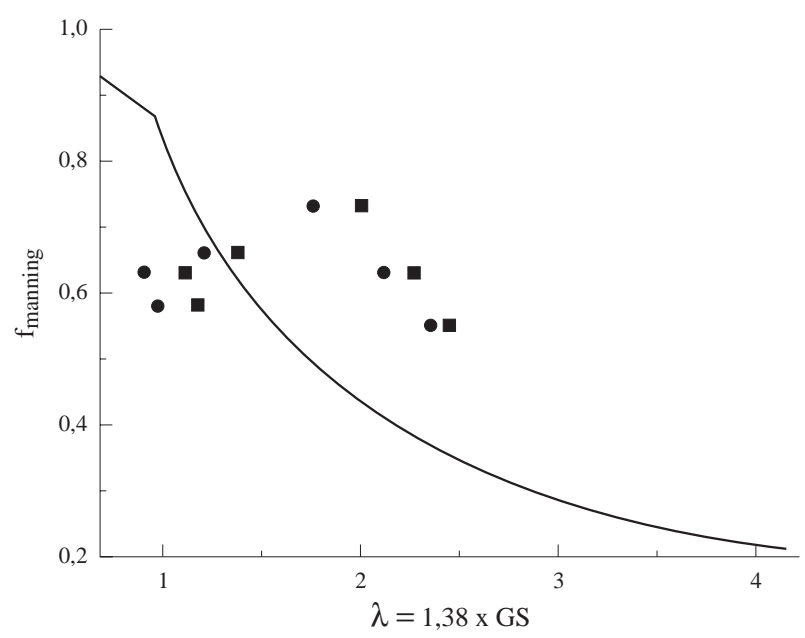

Figura 4. Variação do coeficiente de transporte $\left(\mathrm{f}_{\text {Manning }}\right)$ de CMC em função do seu grau médio de substituição $(\overline{G S})$ segundo o modelo de Manning. Valores experimentais determinados por titulações $\left(\overline{G S}_{\mathrm{tit}}: 1\right) \mathrm{e}$ por espectroscopia ${ }^{1} \mathrm{H} \operatorname{rmn}\left(\overline{G S}_{\mathrm{rmn}}: \mathrm{n}\right)$.

A aplicação do modelo de Manning ${ }^{[9]}$ para calcular os coeficientes de transporte ( $f_{\text {manning }}$ ) em função da densidade linear de carga das cadeias de carboximetilcelulose permite prever que, à medida que cadeias mais substituídas são consideradas, seus valores são paulatinamente menores até que o valor limite $\left(\mathrm{f}_{\text {Manning }}=0,21\right)$ é atingido quando $\overline{G S}=3,0$.

A comparação entre as previsões de Manning e os valores experimentais de coeficientes de transporte determinados por titulações condutimétricas não apresentam boa concordância, sejam os valores correspondentes de densidade linear de carga $(\lambda)$ calculados a partir dos graus de substituição determinados por titulação $\left(\overline{\mathrm{GS}}_{\text {tit }}\right)$ ou por espectroscopia ${ }^{1} \mathrm{H} \mathrm{rmn}\left(\overline{G S}_{\mathrm{rmn}}\right)$ (Figura 4).

Essa comparação sugere que as distribuições de substituintes não são uniformes em nenhum dos dois casos, mas também que as amostras menos substituídas de CMC são mais homogeneamente carboximetiladas do que as mais substituídas.

Nos casos das amostras menos substituídas não há uma variação coerente do coeficiente de transporte com o grau médio de substituição, mas os valores experimentais e previstos são mais próximos. Por outro lado, os valores de coeficientes de transporte experimentais e calculados das amostras com $\overline{G S}>1,0$ são menos semelhantes. Entretanto, a tendência de decréscimo do coeficiente de transporte com o aumento de $\overline{G S}$, como teoricamente previsto, é obedecida.

O desacordo quantitativo entre os valores previstos e os resultados experimentais pode ser em parte atribuído à natureza associativa do polímero mas também à inadequação do modelo.
De fato, é atualmente reconhecido que algumas das principais premissas do modelo de Manning não são aplicáveis a polieletrólitos em um amplo intervalo de densidade linear de carga e, além disso, sua aplicação fornece previsões mais coerentes quando macromoléculas rígidas são consideradas ${ }^{[21]}$.

Dentre as considerações questionáveis do modelo de Manning, as seguintes são as mais importantes:

i) o potencial eletrostático médio, adotado para descrever a distribuição de contra-íons em torno do polieletrólito, não leva em conta a flutuação desses contra-íons e seu efeito sobre as propriedades conformacionais das cadeias poliméricas;

ii) a simetria esférica do potencial eletrostático, como é proposto através da abordagem de DebyeHückel aplicada a eletrólitos de baixa massa molar e estendida para polieletrólitos, não é adequada para descrever as vizinhanças de cadeias poliméricas carregadas, a não ser as suficientemente rígidas;

iii) a aproximação linearizada da equação de Poisson-Boltzman, adotada para descrever o potencial eletrostático em torno do polieletrólito, subestima a intensidade do campo potencial a que estão sujeitos os contra-íons, mesmo nos casos de cadeias pouco carregadas no limite de diluição infinita.

Especificamente no que diz respeito às previsões de Manning para as propriedades de equilíbrio de carboximetilcelulose preparada por reação heterogênea, uma limitação adicional do modelo empregado pode ser identificada, o que restringe ainda mais sua aplicação a esse sistema.

Esta limitação está ligada ao fato que tal modelo não considera a ocorrência de interações entre cargas cada vez mais próximas ao longo das cadeias (na mesma unidade repetitiva e em unidades adjacentes) e a influência que têm sobre as propriedades do polieletrólito.

\section{Conclusões}

A ocorrência de processos associativos, que levam à formação de agregados mais ou menos estáveis, pode justificar os desacordos entre os valores de graus de substituição de CMC determinados através de titulações e por espectroscopia ${ }^{1} \mathrm{H} \mathrm{rmn}$, e explicar as diferenças entre amostras mais e menos substituídas.

A estabilidade dos agregados formados depende da natureza associativa das interações dos grupos en- 
volvidos (-COOH e -OH), de sua distribuição e de seu número, da taxa com que essas interações são quebradas e reconstituídas e, certamente, das repulsões entre grupos carregados $\left(-\mathrm{COO}^{(-)}\right)$.

No caso das amostras de CMC pouco substituídas, devido ao pequeno número de grupos substituintes, pode ser concluído que as interações eletrostáticas repulsivas são minimizadas e que a conformação enovelada das cadeias é favorecida, propiciando muitas interações associativas que são quebradas e reconstituídas rapidamente nos microdomínios em que ocorrem. A ocorrência desses intercruzamentos reversíveis aumenta a atração efetiva entre segmentos poliméricos, reduzindo sua afinidade pelo solvente e favorecendo a agregação ${ }^{[8]}$.

Assim, a distribuição em blocos curtos, pouco numerosos e relativamente espaçados de grupos carboximetila ao longo das cadeias dessas amostras menos substituídas, como discutido, deve ser um fator muito importante para estabilizar tais interações associativas.

Para as amostras mais substituídas, o padrão de substituição também corresponde a blocos de grupos carboximetilas que, no entanto, vão se tornando mais longos e menos espaçados à medida que $\overline{G S}$ aumen$\operatorname{ta}^{[3,4,12]}$. Nesses casos um efeito de volume excluído eletrostático, devido à presença de mais grupos iônicos ao longo das cadeias, contribui para que uma conformação mais estendida seja adotada, o que desfavorece a ocorrência de interações associativas e a estabilidade dos agregados formados. Assim, embora as cadeias poliméricas das amostras mais substituídas de CMC conservem parte de sua capacidade de interagir associativamente, as estruturas agregadas são menos estabilizadas e, portanto, mais facilmente dissociadas. Devido ao menor grau de agregação em solução, as determinações de $\overline{G S}$ por titulações e através de espectroscopia ${ }^{1} \mathrm{H} \mathrm{rmn}$ dessas amostras apresentam melhor concordância que nos casos das amostras menos substituídas.

De maneira geral, as limitações do modelo de Manning e o fenômeno de agregação/associação de cadeias de CMC podem ser os fatores responsáveis pelas discordâncias entre os resultados experimentais obtidos com diferentes métodos analíticos e também pelo desacordo entre as previsões teóricas e os valores experimentais de coeficientes de transporte.

\section{Agradecimentos}

Os autores agradecem ao $\mathrm{CNPq}$ pela bolsa de
Doutorado concedida a José Cláudio Caraschi e à FAPESP pelo apoio financeiro outorgado durante a realização deste trabalho.

\section{Referências bibliográficas}

1. Juste, K.E.; Majewicz, T.G.; "Cellulose ethers", in: Encyclopedia of Polymer Science and Engineering 2.ed., v.3, p.226-269; Kroschwitz, J. I. ed.; New York, John Wiley \& Sons (1985).

2. Yalpani, M.; "Polysaccharides Synthesis, Modifications and Structure/Property Relations", cap.1, p.1-7; Amsterdam, Elsevier (1988).

3. Heinze, T.; Erler, U.; Nehls, I.; Klemm, D.; Ang. Makromol. Chem. 215, p. 93-106 (1994).

4. Caraschi, J.C.; Rocha, G.J.M.; Campana Filho, S.P.; "Determination of the substituent pattern of carboxymethylcellulose by using highperformance liquid chromatography", in: Proceedings of the Fifth Brazilian Symposium on the Chemistry of Lignins and Other Wood Components, p. 99-106, Curitiba - PR, ago/set (1997).

5. Tezuka, Y.; Tsuchiya,Y.; Shiomi, T.; Carbohydr. Res. 291, 99-108 (1996).

6. Schulz, L.; Burchard, W.; Dönges, R.; "Evidence of supramolecular structures of cellulose derivatives in solution", in: Cellulose Derivatives: modification, characterization, and nanostructures, cap.16, p.218-238; Thomas J. Heinze \& Wolfang G. Glasser (eds.); American Chemical Society, Washington (1996).

7. Liebert, T.; Heinze, T.; "Induced phase separation: a new synthesis concept in cellulose chemistry", in: Cellulose Derivatives: modification, characterization, and nanostructures, cap.4, p.61-72; Thomas J. Heinze \& Wolfang G. Glasser eds.; American Chemical Society, Washington (1996).

8. Rubinstein, M.; Dobrynin, A.V.; TRIP 5, p.181 (1997).

9. Manning, G.S.; J. Chem. Phys. 51, p. 924 (1969).

10. Caraschi, J.C. - "Estudo das relações estrutura/ propriedades de carboximetilcelulose obtida por 
derivatização de polpa de bagaço de cana-deaçúcar", Tese de Doutorado, Universidade de São Paulo, Brasil (1997).

11. Caraschi, J.C.; Campana Filho, S.P.; "On the preparation and characterization of carboxymethylcellulose from bleached soda/ anthraquinone pulp of sugar cane bagasse", in: Proceedings of the Second International Symposium on Natural Polymers and Composites, p. 163-166, Atibaia - SP, mai (1998).

12. Ho, F.F.L.; Klosiewicz, D.W.; Anal. Chem. 52, p.913-916 (1980).

13. Rinaudo M.; Hundry-Clergeon, G.; J. Chim. Phys. Phys.-Chim. Biol. 64, p.1746 (1967).

14. Lifson, S.; Katachalsky, A.; J. Polym. Sci. 13, p.43-45 (1954).

15. Rinaudo, M.; "Comparison between experimental results obtained with hydroxylated polyacids and some theoretical models" in: Polyelectrolytes; p.157-193; D. Reidel Publishing Company, Dordrecht-Holland (1974).
16. Milas, M.; "Polieletrólitos", p.42-45; R.A.M.C. Groote e A.A.S. Curvelo ed.; Universidade de São Paulo (1991).

17. Dautzenberg, H.; Jaeger, W.; Kötz, J.; Philipp, B.; Seidel, Ch.; Stscherbina, D.; "Electrochemical and spectroscopic characterization of polyelectrolytes", em: Polyelectrolytes formation, characterization and application, cap.4, p.133; Hanser Publishers, Munich (1994).

18. Baar, A.; Kulicke, W.M.; Szablikowski, W.; Kiesewetter, R.; Macromol. Chem. Phys. 195, p.1483 (1994).

19. Kitano, T.; Kawaguchi, S.; Ito, K.; Minataka, A.; Macromolecules 20, p. 1598 (1987).

20. Martinez, C.M.F.; Frollini, E.; Campana Filho, S.P.; Polímeros, Ciência e Tecnologia, p.48 (1997).

21. Dautzenberg, H.; Jaeger, W.; Kötz, J.; Philipp, B.; Seidel, Ch.; Stscherbina, D.; "Polyelectrolyte models and theroretical predictions", em: Polyelectrolytes formation, characterization and application, cap.3, Hanser Publishers, Munich (1994).

Recebido em: 04/01/99 Aprovado em: 10/06/99 